\title{
Higher order Glaeser inequalities and optimal regularity of roots of real functions
}

\author{
MARINA GHISI AND MASSIMO GOBBINO
}

Dedicated to Professor Sergio Spagnolo on the occasion of his 70 th birthday

\begin{abstract}
We prove a higher order generalization of the Glaeser inequality, according to which one can estimate the first derivative of a function in terms of the function itself and the Hölder constant of its $k$-th derivative.

We apply these inequalities in order to obtain pointwise estimates on the derivative of the $(k+\alpha)$-th root of a function of class $C^{k}$ whose derivative of order $k$ is $\alpha$-Hölder continuous. Thanks to such estimates, we prove that the root is not just absolutely continuous, but its derivative has a higher summability exponent.

Some examples show that our results are optimal.
\end{abstract}

Mathematics Subject Classification (2010): 26A46 (primary); 26B30, 26A27 (secondary).

\section{Introduction}

Let $v \in C^{2}(\mathbb{R})$ be a function such that either $v(x) \geq 0$ or $v(x) \leq 0$ for every $x \in \mathbb{R}$. Let us assume that $v^{\prime \prime}(x)$ is bounded. Then one has that

$$
\left|v^{\prime}(x)\right|^{2} \leq 2|v(x)| \cdot \sup _{x \in \mathbb{R}}\left|v^{\prime \prime}(x)\right| \quad \forall x \in \mathbb{R} .
$$

This is known as Glaeser inequality (see [7]). If we set $u(x):=|v(x)|^{1 / 2}$, then estimate (1.1) implies that

$$
\left|u^{\prime}(x)\right|=\frac{\left|v^{\prime}(x)\right|}{2|v(x)|^{1 / 2}} \leq\left\{\frac{1}{2} \sup _{x \in \mathbb{R}}\left|v^{\prime \prime}(x)\right|\right\}^{1 / 2}
$$

for every $x \in \mathbb{R}$ such that $v(x) \neq 0$. In other words, a bound on the second derivative of some function $v(x)$ with constant sign yields a bound on the first derivative of the square root of $|v(x)|$.

Received July 28, 2011; accepted in revised form January 16, 2012. 
The first question we address in this paper is how to obtain bounds on the first derivative of higher order roots of $v$. We prove that for every integer $k \geq 2$ there exists a constant $C(k)$, depending only upon $k$, such that

$$
\left|v^{\prime}(x)\right|^{k+1} \leq C(k) \cdot|v(x)|^{k} \cdot \sup _{x \in \mathbb{R}}\left|v^{(k+1)}(x)\right| \quad \forall x \in \mathbb{R}
$$

for every $v \in C^{k+1}(\mathbb{R})$ such that either $v(x) \geq 0$ or $v(x) \leq 0$ for every $x \in \mathbb{R}$, and either $v^{\prime}(x) \geq 0$ or $v^{\prime}(x) \leq 0$ for every $x \in \mathbb{R}$. This means that we can estimate the first derivative of $u(x):=|v(x)|^{1 /(k+1)}$ (even without absolute value, if $k$ is even) in terms of the $(k+1)$-th derivative of $v$, namely

$$
\left|u^{\prime}(x)\right|=\frac{1}{k+1} \cdot \frac{\left|v^{\prime}(x)\right|}{|v(x)|^{k /(k+1)}} \leq \frac{1}{k+1}\left\{C(k) \sup _{x \in \mathbb{R}}\left|v^{(k+1)}(x)\right|\right\}^{1 /(k+1)}
$$

for every $x \in \mathbb{R}$ such that $v(x) \neq 0$. We refer to Theorem 2.1 below for a slightly more general result. Example 4.1 and Example 4.2 below show the optimality of the assumptions. It is quite interesting that for $k \geq 2$ we have to impose that also $v^{\prime}(x)$ does not change its sign, but we do not need similar assumptions on higher order derivatives (even if such assumptions would strongly simplify proofs).

The second question we address in this paper is to obtain similar estimates for the first derivative of roots of a function defined in some interval $(a, b)$. So we take a function $g:(a, b) \rightarrow \mathbb{R}$, an integer $k \geq 1$, and any continuous function $f:(a, b) \rightarrow \mathbb{R}$ such that

$$
|f(x)|^{k+1}=|g(x)| \quad \forall x \in(a, b) .
$$

Trivial examples suggest that without assumptions on the sign of $g$ we cannot expect more than some sort of absolute continuity of $f$.

This problem was considered for the first time in [4, Lemma 1], where the authors proved that $f$ is absolutely continuous in $(a, b)$ provided that $g \in C^{k+1}((a, b))$ is a nonnegative function. This is a quite strong requirement which, for example, forces $g^{\prime}$ to vanish whenever $g$ vanishes. More recently, S. Tarama [13] extended the technique introduced in [4] and proved the same conclusion without assuming that $g$ is nonnegative. We state Tarama's result in Section 2 as Theorem A.

The absolute continuity of $f$ is equivalent to saying that $f^{\prime} \in L^{1}((a, b))$. In this paper we prove (Theorem 2.2) a better summability of $f^{\prime}$, namely that $f^{\prime} \in$ $L^{p}((a, b))$ for every $p<1+1 / k$. Example 4.3 and Example 4.4 below show the optimality of our result, both with respect to $p$, and with respect to the regularity of $g$. Although the result is one dimensional, it can be easily extended to any space dimension through a straightforward sectioning argument (see Theorem 2.3).

A higher summability of $f^{\prime}$ was obtained by F. Colombini and N. Lerner in [5], but once again in the case where $g$ is nonnegative. They proved that for every nonnegative $g \in C^{k+1}((a, b))$ one has that $f^{\prime} \in L^{p}((a, b))$ (locally) for every $p<1+2 /(k-1)$, and the same in any space dimension. Of course all solutions 
of (1.3) are also solutions of $|f(x)|^{2 k+2}=|g(x)|^{2}$, and thus their result implies that $f^{\prime} \in L^{p}((a, b))$ for every $p<1+1 / k$ provided that $g \in C^{2 k+2}((a, b))$, without sign restrictions on $g$. In other words, one obtains our summability of $f^{\prime}$ but with twice stronger regularity assumptions on $g$. This is observed in a remark at the end of [5, Section 4]. The authors conclude the same remark by pointing out that it is plausible that the only assumption $g \in C^{k+1}$ is needed in order to obtain the same summability, but the proof of this fact would require a nontrivial modification of their arguments.

In this paper we prove this conjecture, and indeed we follow a completely different path with respect to the previous literature. The technique used in [4] and [13] is oriented to obtaining estimates of the total variation, namely integral estimates. In our approach the higher summability of $f^{\prime}$ follows from suitable pointwise estimates on $f^{\prime}(x)$. To this end, we first divide $(a, b)$ into intervals $\left(a_{i}, b_{i}\right)$ where $g(x) \cdot g^{\prime}(x) \neq 0$. Then in each interval we exploit a natural generalization of Glaeser inequalities (1.2) to functions defined in bounded intervals (see Proposition 3.5). Thanks to this generalization, we have an estimate on $f^{\prime}(x)$, multiplied by a standard cutoff function, in terms of the derivatives of $g$ up to order $(k+1)$, and of the length $\left(b_{i}-a_{i}\right)$ of the interval itself. These estimates yield an upper bound on the measure of the sublevels of $f^{\prime}$ in $\left(a_{i}, b_{i}\right)$. Summing over all subintervals we obtain the conclusion.

We point out that this approach works because the set of points where $g(x)$. $g^{\prime}(x)=0$ does not contribute to the integral of $f^{\prime}(x)$. We stress that this would be false for the set of points where $g(x) \cdot g^{\prime}(x) \cdot g^{\prime \prime}(x)=0$, and this is the reason why it is important to obtain higher order Glaeser inequalities such as (1.2) with assumptions on $v$ and $v^{\prime}$ only.

We conclude by mentioning some related results. Regularity of roots has long been studied, both because the problem is interesting in itself, and because of several applications, ranging from algebraic geometry to partial differential equations, for which we refer to the quoted references.

A first research line concerns square roots, namely the case where $f^{2}=g$ (see [2] and the references quoted therein). The leitmotif of these papers is the search of conditions on $g$ under which one can choose a regular enough root $f$. The usual requirement is the existence of sufficiently many derivatives of $g$ and their vanishing at the zeroes of $g$.

Our problem is different for two reasons. Firstly we investigate the regularity of a given root, which we cannot choose. Secondly, we have no assumptions on the sign of $g$, since we are actually solving $|f|^{k+1}=|g|$.

Roots of functions are just the first step toward roots of polynomials with coefficients depending on a parameter. Regularity results for roots of nonnegative functions have their counterpart in analogous results for roots of hyperbolic polynomials, namely polynomials whose roots are all real. Classical conclusions are that one can choose roots of class $C^{1}$ or twice differentiable provided that coefficients are smooth enough (see $[1,3,6,9,10,14,15]$ ).

The situation considered in this paper corresponds to non-hyperbolic polynomials, in which case one investigates the regularity of a given continuous root. The 
problem is still quite open. T. Kato [8] proved that for a monic polynomial of degree $n$ with continuous coefficients one can always choose $n$ continuous functions representing the roots of the polynomial. S. Spagnolo [12] proved that continuous roots of monic polynomials of degree 2 and 3 are locally absolutely continuous provided that coefficients are of class $C^{5}$ and $C^{25}$, respectively. This result is based on the explicit formulae for solutions, and on the nonoptimal [4, Lemma 1], and for this reason it is probably nonoptimal.

Spagnolo's result was in some sense extended by A. Rainer [11], who proved the absolute continuity of roots of monic polynomials of arbitrary degree with coefficients of class $C^{\infty}$, but with the assumption that one can continuously arrange roots in such a way that no two of them meet with an infinite order of flatness. In the case of the polynomial $P(y):=y^{k}-g(x)$, namely in the case of $k$-th roots, this implies that $g$ has a finite number of zeroes. This simplifying assumption greatly reduces the difficulty of the problem, but the estimates obtained in this way do blow up when the number of zeroes goes to infinity. In this paper we do not impose this simplifying assumption, and indeed the potential accumulation of zeroes of $g$ and its derivatives is the main difficulty we have to face in our proofs.

We hope that our new approach based on pointwise estimates could be helpful in order to understand in full generality the case of non-hyperbolic polynomials.

This paper is organized as follows. In Section 2 we fix notations, and we state our main results. In Section 3 we prove them. In Section 4 we present the examples showing the optimality of our results, and the open problem concerning the regularity of roots of non-hyperbolic polynomials.

ACKNOWLEDGEMENTS. We discovered this problem thanks to a conference held by Prof. F. Colombini during the meeting "Asymptotic Properties of Solutions to Hyperbolic Equations" (London, March 2011). For this reason, we are indebted to him and to the organizers of that workshop.

We would like also to thank Prof. S. Spagnolo for pointing out reference [13], and Prof. F. Colombini for pointing out reference [5].

Finally, we thank the anonymous referee for carefully checking the manuscript, and for many insightful and powerful suggestions which greatly improved the clarity of the presentation.

\section{Notation and statements}

Let $\Omega \subseteq \mathbb{R}$ be an open set, and let $\alpha \in(0,1]$. A function $f: \Omega \rightarrow \mathbb{R}$ is called $\alpha$-Hölder continuous if there exists a constant $H$ such that

$$
|f(y)-f(x)| \leq H|y-x|^{\alpha} \quad \forall(x, y) \in \Omega^{2} .
$$


In this case

$$
\operatorname{Höld}_{\alpha}(f, \Omega):=\sup \left\{\frac{|f(y)-f(x)|}{|y-x|^{\alpha}}:(x, y) \in \Omega^{2}, x \neq y\right\}
$$

denotes the $\alpha$-Hölder constant of $f$ in $\Omega$, namely the smallest $H$ for which (2.1) holds true. Note that this includes the case of Lipschitz continuous functions $(\alpha=1)$.

If $k$ is a positive integer, $C^{k, \alpha}(\Omega)$ denotes the set of functions $g \in C^{k}(\Omega)$ with derivatives up to order $k$ which are bounded in $\Omega$, and whose $k$-th derivative $g^{(k)}$ is $\alpha$-Hölder continuous in $\Omega$.

Regularity of roots of elements of $C^{k, \alpha}$ was investigated in [13]. The precise result is the following.

Theorem A. Let $k$ be a positive integer, let $\alpha \in(0,1]$, let $(a, b) \subseteq \mathbb{R}$ be an interval, and let $f:(a, b) \rightarrow \mathbb{R}$ and $g:(a, b) \rightarrow \mathbb{R}$ be two functions.

Let us assume that $f$ is continuous in $(a, b)$, and that $g \in C^{k, \alpha}((a, b))$ satisfies

$$
|f(x)|^{k+\alpha}=|g(x)| \quad \forall x \in(a, b) .
$$

Then we have that $f^{\prime} \in L^{1}((a, b))$, and there exists a constant $C(k)$ such that

$$
\begin{aligned}
& \mid f^{\prime} \|_{L^{1}((a, b))} \\
& \leq C(k)\left\{\operatorname{Höld}_{\alpha}\left(g^{(k)},(a, b)\right)(b-a)^{k+\alpha}+\sum_{i=1}^{k}\left\|g^{(i)}\right\|_{L^{\infty}((a, b))}(b-a)^{i}\right\}^{1 /(k+\alpha)} .
\end{aligned}
$$

We are now ready to state our main contributions. The first result of this paper is the following generalization of Glaeser inequality.

Theorem 2.1 (Higher order Glaeser inequalities). Let $k$ be a positive integer, let $\alpha \in(0,1]$, and let $v \in C^{k}(\mathbb{R})$ be a function such that $v(x)$ and $v^{\prime}(x)$ do not change their sign in $\mathbb{R}$ (namely either $v(x) \geq 0$ or $v(x) \leq 0$ for every $x \in \mathbb{R}$, and either $v^{\prime}(x) \geq 0$ or $v^{\prime}(x) \leq 0$ for every $\left.x \in \mathbb{R}\right)$.

Let us assume that the $k$-th derivative $v^{(k)}(x)$ is $\alpha$-Hölder continuous in $\mathbb{R}$.

Then there exists a constant $C(k)$, depending only upon $k$, such that

$$
\left|v^{\prime}(x)\right|^{k+\alpha} \leq C(k) \cdot|v(x)|^{k+\alpha-1} \cdot \operatorname{Höld}_{\alpha}\left(v^{(k)}, \mathbb{R}\right) \quad \forall x \in \mathbb{R} .
$$

A careful inspection of the proof of Theorem 2.1 reveals that in the case $k=1$ we do not need assumptions on the sign of $v^{\prime}(x)$, as in the standard Glaeser inequality (1.1).

Theorem 2.1 above is optimal. Indeed

- in Example 4.1 we construct a positive function $v \in C^{\infty}(\mathbb{R})$, whose derivatives are all bounded, and such that the ratio

$$
\frac{\left|v^{\prime}(x)\right|^{k+\alpha}}{[v(x)]^{k+\alpha-1}}
$$


is unbounded for every $k \geq 2$ and every $\alpha>0$ (of course $v^{\prime}(x)$ is a sign changing function),

- in Example 4.2 we construct a function $v \in C^{\infty}(\mathbb{R})$ such that the product $v(x)$. $v^{\prime}(x)$ never vanishes, $v \in C^{k, \beta}(\mathbb{R})$ for every $\beta<\alpha$, and the ratio (2.4) is unbounded.

In the second result we consider functions defined on an interval $(a, b)$, and we improve Theorem A by showing a better summability of $f^{\prime}$. As in [5] we express this better summability in terms of weak $L^{p}$ spaces, whose definition we briefly recall.

If $\Omega \subseteq \mathbb{R}$ is a bounded open set, and $\psi: \Omega \rightarrow \mathbb{R}$ is a measurable function, then for every $p \geq 1$ one can set

$$
\|\psi\|_{p, w, \Omega}:=\sup _{M \geq 0}\left\{M \cdot[\operatorname{meas}\{x \in \Omega:|\psi(x)|>M\}]^{1 / p}\right\},
$$

where "meas" denotes the Lebesgue measure. The weak $L^{p}$ space $L_{w}^{p}(\Omega)$ is the set of all functions for which this quantity (which is not a norm, since the triangular inequality fails to hold) is finite. In Lemma 3.1 we state all the properties of weak $L^{p}$ spaces needed in this paper. Here we just recall that for every $q<p$ we have that $L^{p}(\Omega) \subset L_{w}^{p}(\Omega) \subset L^{q}(\Omega)$, with strict inclusions.

We are now ready to state our main result.

Theorem 2.2 (Optimal regularity of roots). Let $k$ be a positive integer, let $\alpha \in$ $(0,1]$, let $(a, b) \subseteq \mathbb{R}$ be an interval, and let $f:(a, b) \rightarrow \mathbb{R}$ be a function.

Let us assume that $f$ is continuous in $(a, b)$, and there exists $g \in C^{k, \alpha}((a, b))$ such that (2.2) holds true. Let $p=p(k, \alpha)$ be such that

$$
\frac{1}{p}+\frac{1}{k+\alpha}=1 \text {. }
$$

Then we have that $f^{\prime} \in L_{w}^{p}((a, b))$, and there exists a constant $C(k)$, depending only upon $k$, such that

$$
\begin{aligned}
& \left\|f^{\prime}\right\|_{p, w,(a, b)} \\
& \leq C(k) \max \left\{\left[\operatorname{Höld}_{\alpha}\left(g^{(k)},(a, b)\right)\right]^{1 /(k+\alpha)} \cdot(b-a)^{1 / p},\left\|g^{\prime}\right\|_{L^{\infty}((a, b))}^{1 /(k+\alpha)}\right\} .
\end{aligned}
$$

In particular, we have that $f$ lies in the Sobolev space $W^{1, q}((a, b))$ for every $q \in$ $[1, p)$.

We point out that in the right-hand side of (2.6) we have the maximum between two terms which are homogeneous of degree $1-1 / p$ with respect to dilatations (namely when replacing $g(x)$ with $g(\lambda x)$ ). Also the left-hand side of (2.6) is homogeneous with the same degree. Moreover Theorem 2.2 above is optimal, in the sense that

- in Example 4.3 below we construct $f$ and $g$ such that $g \in C^{k, \alpha}((a, b)) \cap$ $C^{\infty}((a, b))$, but $f^{\prime} \notin L^{p}((a, b))$ for $p$ given by $(2.5)$, 
- in Example 4.4 below we construct $f$ and $g$ such that $g \in C^{\infty}((a, b))$ and $g \in C^{k, \beta}((a, b))$ for every $\beta<\alpha$, but $f$ is not a bounded variation function in $(a, b)$, hence also $f^{\prime} \notin L^{1}((a, b))$.

Example 4.4 shows also that there is some sort of gap phenomenon. If $g \in C^{k, \beta}$ for all $\beta<\alpha$, then it is possible that its $(k+\alpha)$-th root $f$ is not even a bounded variation function. As soon as $g \in C^{k, \alpha}$ we have that $f \in W^{1, q}$ for every $q \in[1, p)$. So we have an immediate jump in the regularity of the root without passing through intermediate values of the Sobolev exponent.

We conclude by extending Theorem 2.2 to higher dimension. The extension is straightforward because the spaces $C^{k, \alpha}(\Omega)$ and $L_{w}^{p}(\Omega)$ can be easily defined for every open set $\Omega \subseteq \mathbb{R}^{n}$, and the Sobolev regularity of a function of $n$ real variables depends on the Sobolev regularity of its one-dimensional sections.

We obtain the following result.

Theorem 2.3 (Higher dimensional case). Let $n$ and $k$ be two positive integers, let $\alpha \in(0,1]$, let $\Omega \subseteq \mathbb{R}^{n}$ be an open set, and let $f: \Omega \rightarrow \mathbb{R}$ be a function.

Let us assume that $f$ is continuous in $\Omega$, and there exists $g \in C^{k, \alpha}(\Omega)$ such that (2.2) holds true for every $x \in \Omega$. Let $p$ be defined by (2.5), and let $\operatorname{Höld}_{\alpha}\left(g^{(k)}, \Omega\right)$ be the maximum of the Hölder constants in $\Omega$ of all partial derivatives of $g$ with order $k$.

Then $\nabla f \in L_{w}^{p}\left(\Omega^{\prime}\right)$ for every $\Omega^{\prime} \subset \subset \Omega$, and there exist constants $C_{1}\left(n, k, \Omega, \Omega^{\prime}\right)$ and $C_{2}\left(n, k, \Omega, \Omega^{\prime}\right)$, both independent of $f$, such that

$$
\begin{aligned}
& \|\nabla f\|_{p, w, \Omega^{\prime}} \\
& \leq \max \left\{C_{1}\left(n, k, \Omega, \Omega^{\prime}\right)\left[\operatorname{Höld}_{\alpha}\left(g^{(k)}, \Omega\right)\right]^{1 /(k+\alpha)}, C_{2}\left(n, k, \Omega, \Omega^{\prime}\right)\|\nabla g\|_{L^{\infty}(\Omega)}^{1 /(k+\alpha)}\right\} .
\end{aligned}
$$

A careful inspection of the proof reveals that $C_{1}$ and $C_{2}$ have homogeneity degrees with respect to dilatations equal to $-n / p$ and $-(n-1) / p$, respectively, which makes the two terms in the maximum homogeneous with the same degree of the left-hand side. It also reveals that what we actually need to assume on $g$ is the regularity of its one-dimensional sections obtained by freezing $(n-1)$ variables in all possible ways.

\section{Proofs}

\subsection{Technical preliminaries}

Lemma 3.1 (Properties of weak $L^{p}$ spaces). For every bounded open set $\Omega \subseteq \mathbb{R}$, and every $p \geq 1$, we have the following conclusions.

(1) If $\psi_{1}, \ldots, \psi_{n}$ are elements of $L_{w}^{p}(\Omega)$, then

$$
\left\|\psi_{1}+\ldots+\psi_{n}\right\|_{p, w, \Omega} \leq n\left(\left\|\psi_{1}\right\|_{p, w, \Omega}+\ldots+\left\|\psi_{n}\right\|_{p, w, \Omega}\right) .
$$


(2) We have that

$$
\|\lambda \psi\|_{p, w, \Omega}=|\lambda| \cdot\|\psi\|_{p, w, \Omega} \quad \forall \lambda \in \mathbb{R}, \forall \psi \in L_{w}^{p}(\Omega) .
$$

(3) For every $\psi_{1}$ and $\psi_{2}$ in $L_{w}^{p}(\Omega)$ we have that

$$
\left\|\max \left\{\psi_{1}, \psi_{2}\right\}\right\|_{p, w, \Omega} \leq 2 \max \left\{\left\|\psi_{1}\right\|_{p, w, \Omega},\left\|\psi_{2}\right\|_{p, w, \Omega}\right\} .
$$

(4) Let $\Omega:=(a, b)$ be an interval, andlet $\psi_{1}(x):=1$ and $\psi_{2}(x):=[(x-a)(b-x)]^{-1 / p}$ for every $x \in(a, b)$. Then we have that

$$
\left\|\psi_{1}\right\|_{p, w,(a, b)}=(b-a)^{1 / p}, \quad\left\|\psi_{2}\right\|_{p, w,(a, b)} \leq\left(\frac{4}{b-a}\right)^{1 / p} .
$$

(5) Let $\left\{\Omega_{J}\right\}_{J \in \mathcal{C}}$ be a finite or countable family of open sets whose union is $\Omega$. Then we have that

$$
\|\psi\|_{p, w, \Omega}^{p} \leq \sum_{J \in \mathcal{C}}\|\psi\|_{p, w, \Omega_{J}}^{p} \quad \forall \psi \in L_{w}^{p}(\Omega) .
$$

Proof. All these properties are simple consequences of the definition of weak $L^{p}$ spaces. In order to estimate $\left\|\psi_{2}\right\|_{p, w,(a, b)}$ in statement (4) it is enough to remark that

$$
\begin{aligned}
\operatorname{meas}\left\{x \in(a, b):\left|\psi_{2}(x)\right|>M\right\} & =\operatorname{meas}\left\{x \in(a, b):(x-a)(b-x)<M^{-p}\right\} \\
& \leq 2 \operatorname{meas}\left\{x \in\left(a, \frac{a+b}{2}\right):(x-a)\left(\frac{b-a}{2}\right)<\frac{1}{M^{p}}\right\} \\
& \leq \frac{4}{b-a} \cdot \frac{1}{M^{p}} .
\end{aligned}
$$

Lemma 3.2. Let $(a, b) \subseteq \mathbb{R}$ be an interval, let $f:(a, b) \rightarrow \mathbb{R}$ be a continuous function, and let $\Omega_{0}:=\{x \in(a, b): f(x) \neq 0\}$.

Let us assume that $f \in C^{1}\left(\Omega_{0}\right)$, and that $f^{\prime} \in L_{w}^{p}\left(\Omega_{0}\right)$ for some $p>1$.

Then we have that the distributional derivative of $f$ in $(a, b)$ is a measurable function $f^{\prime} \in L_{w}^{p}((a, b))$, and

$$
\left\|f^{\prime}\right\|_{p, w,(a, b)}=\left\|f^{\prime}\right\|_{p, w, \Omega_{0}} .
$$

Proof. Let us set

$$
\psi(x):= \begin{cases}f^{\prime}(x) & \text { if } x \in \Omega_{0} \\ 0 & \text { if } x \in(a, b) \backslash \Omega_{0} .\end{cases}
$$

Clearly we have that $\psi \in L_{w}^{p}((a, b))$. We claim that $\psi$ is the distributional derivative of $f$ in $(a, b)$. Indeed let us take any compactly supported test function $\phi \in$ $C^{\infty}((a, b))$. Let $\mathcal{C}$ denote the (finite or countable) set of connected components of 
$\Omega_{0}$. Then in each $J \in \mathcal{C}$ we can use the standard integration-by-parts formula for smooth functions, and obtain that

$$
\begin{aligned}
\int_{a}^{b} f(x) \phi^{\prime}(x) d x & =\int_{\Omega_{0}} f(x) \phi^{\prime}(x) d x=\sum_{J \in \mathcal{C}} \int_{J} f(x) \phi^{\prime}(x) d x \\
& =-\sum_{J \in \mathcal{C}} \int_{J} f^{\prime}(x) \phi(x) d x=-\int_{\Omega_{0}} f^{\prime}(x) \phi(x) d x \\
& =-\int_{a}^{b} \psi(x) \phi(x) d x
\end{aligned}
$$

Now that we know that $\psi$ is the distributional derivative of $f$ it is easy to conclude that

$$
\left\|f^{\prime}\right\|_{p, w,(a, b)}=\|\psi\|_{p, w,(a, b)}=\|\psi\|_{p, w, \Omega_{0}}=\left\|f^{\prime}\right\|_{p, w, \Omega_{0}},
$$

which completes the proof.

\subsection{Key estimates}

The following surprising property of polynomials is the key tool in the proof of our higher order Glaeser inequalities. We stress that the "magic constants" $\delta_{k}$ and $\varepsilon_{k}$ do not depend on $P(x)$.

Lemma 3.3 (Magic constants). For every positive integer $k$ there exist positive constants $\delta_{k}$ and $\varepsilon_{k}$, depending only upon $k$, with the following properties.

(1) For every polynomial $P(x)$, with degree less than or equal to $k$, such that $P(0)=1$ and $P^{\prime}(0)=-1$, there exists $x \in\left[0, \delta_{k}\right]$ such that either $P(x) \leq-1$ or $P^{\prime}(x) \geq 1$.

(2) For every real number $A$, every $\alpha \in(0,1]$, and every polynomial $P(x)$, with degree less than or equal to $k$, such that $P(0)=1$ and $P^{\prime}(0)=-1$, the following implication

$$
\begin{array}{ll}
P(x)+A x^{k+\alpha} \geq 0 & \forall x \in\left[0, \delta_{k}\right] \\
P^{\prime}(x)-A k x^{k+\alpha-1} \leq 0 & \forall x \in\left[0, \delta_{k}\right]
\end{array} \Longrightarrow \quad A \geq \varepsilon_{k}
$$

holds true.

Proof. Let us assume that statement (1) is false for some $k$. Then there exists a sequence of polynomials $P_{n}(x)$, with degree less than or equal to $k$, such that $P_{n}(0)=1, P_{n}^{\prime}(0)=-1$, and

$$
P_{n}(x) \geq-1 \quad \text { and } \quad P_{n}^{\prime}(x) \leq 1 \quad \forall x \in[0, n] .
$$

These assumptions easily imply that

$$
-1 \leq P_{n}(x) \leq P_{n}(0)+x=1+x \leq 2
$$


for every $x \in[0,1]$. In particular, the sequence $\left\{P_{n}(x)\right\}$ is bounded for at least $(k+1)$ distinct values of $x$, which we denote by $x_{1}, \ldots, x_{k}, x_{k+1}$.

As a consequence, there exists a polynomial $P_{\infty}(x)$, with degree at most $k$, such that $P_{n}(x) \rightarrow P_{\infty}(x)$ up to subsequences (not relabeled), in the sense that we have both convergence of coefficients, and pointwise convergence. This is a well known fact which follows, for example, from the simple remark that coefficients of a polynomial $P(x)$ with degree less than or equal to $k$ depend in a linear way (through a Vandermonde matrix) from the values of $P(x)$ in the $(k+1)$ points $x_{1}$, $\ldots, x_{k}, x_{k+1}$. Alternatively, it follows from the fact that the maximum of $\left|P\left(x_{i}\right)\right|$ for $i=1, \ldots, k+1$, and the maximum of the absolute value of the coefficients of $P(x)$ are two norms on the space of all polynomials with degree less than or equal to $k$, and they are equivalent because the space is finite dimensional.

In any case, convergence of coefficients implies that $P_{\infty}(0)=1$ and $P_{\infty}^{\prime}(0)=$ -1 . Pointwise convergence implies that we can pass to the limit in (3.3) and obtain that

$$
P_{\infty}(x) \geq-1 \quad \text { and } \quad P_{\infty}^{\prime}(x) \leq 1 \quad \forall x \geq 0 .
$$

If the degree of $P_{\infty}(x)$ is 1 , then $P_{\infty}(x)=1-x$, which does not satisfy (3.4). If the degree of $P_{\infty}(x)$ is at least 2, then as $x \rightarrow+\infty$ we have that both $P_{\infty}(x)$ and $P_{\infty}^{\prime}(x)$ tend to $+\infty$ or $-\infty$ according to the sign of the leading coefficient. This contradicts (3.4) also in this case, and completes the proof of statement (1).

Now we claim that the conclusion of statement (2) is true with

$$
\varepsilon_{k}:=\min \left\{\frac{1}{\delta_{k}^{k+1}}, \frac{1}{k \delta_{k}^{k}}\right\}
$$

Indeed from statement (1) we know that there exists $x \in\left[0, \delta_{k}\right]$ such that either $P(x) \leq-1$ or $P^{\prime}(x) \geq 1$. In the first case the first inequality in the left-hand side of (3.2) gives that (clearly $\delta_{k} \geq 1$ for every $k \geq 1$ )

$$
A \geq \frac{-P(x)}{x^{k+\alpha}} \geq \frac{1}{x^{k+\alpha}} \geq \frac{1}{\delta_{k}^{k+\alpha}} \geq \frac{1}{\delta_{k}^{k+1}} .
$$

In the second case the second inequality in the left-hand side of (3.2) gives that

$$
A \geq \frac{P^{\prime}(x)}{k x^{k+\alpha-1}} \geq \frac{1}{k x^{k+\alpha-1}} \geq \frac{1}{k \delta_{k}^{k+\alpha-1}} \geq \frac{1}{k \delta_{k}^{k}} .
$$

In both cases implication (3.2) holds true.

Next result is the main step toward higher order Glaeser inequalities, both in $\mathbb{R}$ and in bounded intervals. The proof exploits the "magic constants" of Lemma 3.3 and Taylor's expansion. 
Proposition 3.4 (Higher order Glaeser inequality in an interval). Let $k$ be a positive integer, and let $\alpha \in(0,1], x_{0} \in \mathbb{R}$, and $d>0$ be real numbers.

Let $v \in C^{k}\left(\left(x_{0}-d, x_{0}+d\right)\right)$ be a function such that $v(x)$ and $v^{\prime}(x)$ do not change their sign, namely either $v(x) \geq 0$ or $v(x) \leq 0$ for every $x \in\left(x_{0}-d, x_{0}+d\right)$, and the same for $v^{\prime}(x)$. Let us assume that the $k$-th derivative $v^{(k)}(x)$ is $\alpha$-Hölder continuous in $\left(x_{0}-d, x_{0}+d\right)$.

Then there exists a constant $C(k)$, depending only upon $k$, such that

$$
\begin{aligned}
& \left|v^{\prime}\left(x_{0}\right)\right|^{k+\alpha} \\
& \leq C(k) \cdot\left|v\left(x_{0}\right)\right|^{k+\alpha-1} \cdot \max \left\{\operatorname{Höld}_{\alpha}\left(v^{(k)},\left(x_{0}-d, x_{0}+d\right)\right), \frac{\left|v^{\prime}\left(x_{0}\right)\right|}{d^{k+\alpha-1}}\right\} .
\end{aligned}
$$

Proof. Up to symmetries we can assume, without loss of generality, that $v(x) \geq 0$ and $v^{\prime}(x) \leq 0$ for every $x \in\left(x_{0}-d, x_{0}+d\right)$. If $v^{\prime}\left(x_{0}\right)=0$, then there is nothing to prove. If $v\left(x_{0}\right)=0$, then it is easy to see that also $v^{\prime}\left(x_{0}\right)=0$, and once again there is nothing to prove. So from now on we assume that $v\left(x_{0}\right)>0$ and $v^{\prime}\left(x_{0}\right)<0$.

Let $\delta_{k}$ be the constant of Lemma 3.3. We distinguish two cases.

Case 1. Let us assume that

$$
\frac{\left|v^{\prime}\left(x_{0}\right)\right|}{v\left(x_{0}\right)} d \leq \delta_{k}
$$

This is equivalent to saying that $\left|v^{\prime}\left(x_{0}\right)\right| \leq \delta_{k} d^{-1} v\left(x_{0}\right)$, and hence

$$
\left|v^{\prime}\left(x_{0}\right)\right|^{k+\alpha}=\left|v^{\prime}\left(x_{0}\right)\right| \cdot\left|v^{\prime}\left(x_{0}\right)\right|^{k+\alpha-1} \leq\left|v^{\prime}\left(x_{0}\right)\right| \cdot\left(\frac{\delta_{k}}{d}\right)^{k+\alpha-1}\left[v\left(x_{0}\right)\right]^{k+\alpha-1},
$$

which implies (3.5) in this first case, provided that $C(k) \geq \delta_{k}^{k}$.

Case 2. Let us assume now that

$$
\frac{\left|v^{\prime}\left(x_{0}\right)\right|}{v\left(x_{0}\right)} d>\delta_{k}
$$

Let us set for simplicity $H:=\operatorname{Höld}_{\alpha}\left(v^{(k)},\left(x_{0}-d, x_{0}+d\right)\right)$, and let us write Taylor's expansion of $v(x)$ of order $k$. For every $\delta \in[0, d)$ we obtain that

$$
v\left(x_{0}+\delta\right)=\sum_{j=0}^{k-1} \frac{v^{(j)}\left(x_{0}\right)}{j !} \delta^{j}+\frac{v^{(k)}\left(x_{0}+\xi\right)}{k !} \delta^{k}
$$

for some $\xi \in(0, \delta)$. Since $v^{(k)}$ is $\alpha$-Hölder continuous we have that

$$
\left|v^{(k)}\left(x_{0}+\xi\right)-v^{(k)}\left(x_{0}\right)\right| \leq H \delta^{\alpha} .
$$

Therefore our assumption that $v(x) \geq 0$ for every $x \in\left(x_{0}-d, x_{0}+d\right)$ implies that

$$
0 \leq v\left(x_{0}+\delta\right) \leq \sum_{j=0}^{k} \frac{v^{(j)}\left(x_{0}\right)}{j !} \delta^{j}+\frac{H}{k !} \delta^{k+\alpha} \quad \forall \delta \in[0, d) .
$$


In an analogous way we obtain that

$$
0 \geq v^{\prime}\left(x_{0}+\delta\right) \geq \sum_{j=0}^{k-1} \frac{v^{(j+1)}\left(x_{0}\right)}{j !} \delta^{j}-\frac{H}{(k-1) !} \delta^{k+\alpha-1} \quad \forall \delta \in[0, d) .
$$

Let us write both inequalities with $\delta:=x \cdot v\left(x_{0}\right) \cdot\left|v^{\prime}\left(x_{0}\right)\right|^{-1}$. We obtain that the two inequalities

$$
\begin{aligned}
& 0 \leq\left|v\left(x_{0}\right)\right|\left(\sum_{j=0}^{k} \frac{v^{(j)}\left(x_{0}\right)}{j !} \cdot \frac{\left[v\left(x_{0}\right)\right]^{j-1}}{\left|v^{\prime}\left(x_{0}\right)\right|^{j}} \cdot x^{j}+\frac{H}{k !} \cdot \frac{\left[v\left(x_{0}\right)\right]^{k+\alpha-1}}{\left|v^{\prime}\left(x_{0}\right)\right|^{k+\alpha}} \cdot x^{k+\alpha}\right), \\
& 0 \geq\left|v^{\prime}\left(x_{0}\right)\right|\left(\sum_{j=0}^{k-1} \frac{v^{(j+1)}\left(x_{0}\right)}{j !} \cdot \frac{\left[v\left(x_{0}\right)\right]^{j}}{\left|v^{\prime}\left(x_{0}\right)\right|^{j+1}} \cdot x^{j}-\frac{H}{(k-1) !} \cdot \frac{\left[v\left(x_{0}\right)\right]^{k+\alpha-1}}{\left|v^{\prime}\left(x_{0}\right)\right|^{k+\alpha}} \cdot x^{k+\alpha-1}\right)
\end{aligned}
$$

hold true for every

$$
0 \leq x<\frac{\left|v^{\prime}\left(x_{0}\right)\right|}{v\left(x_{0}\right)} d .
$$

Due to (3.6), the upper bound is larger than $\delta_{k}$, which proves that the two inequalities hold true at least for every $x \in\left[0, \delta_{k}\right]$. Thus if we set

$$
P(x):=\sum_{j=0}^{k} \frac{v^{(j)}\left(x_{0}\right)}{j !} \cdot \frac{\left[v\left(x_{0}\right)\right]^{j-1}}{\left|v^{\prime}\left(x_{0}\right)\right|^{j}} \cdot x^{j}, \quad A:=\frac{H}{k !} \cdot \frac{\left[v\left(x_{0}\right)\right]^{k+\alpha-1}}{\left|v^{\prime}\left(x_{0}\right)\right|^{k+\alpha}},
$$

we have that $P(0)=1, P^{\prime}(0)=-1$, and the assumptions in the left-hand side of (3.2) are satisfied.

Therefore from (3.2) it follows that $A \geq \varepsilon_{k}$, which implies (3.5) also in this second case, provided that $C(k) \geq\left(\varepsilon_{k} k !\right)^{-1}$.

Proof of Theorem 2.1. Let us fix any point $x_{0} \in \mathbb{R}$. Then the assumptions of Proposition 3.4 are satisfied for every $d>0$. The conclusion follows by remarking that

$$
\operatorname{Höld}_{\alpha}\left(v^{(k)},\left(x_{0}-d, x_{0}+d\right)\right) \leq \operatorname{Höld}_{\alpha}\left(v^{(k)}, \mathbb{R}\right),
$$

and finally letting $d \rightarrow+\infty$.

\subsection{Absolute continuity of roots}

The aim of this section is to prove Theorem 2.2 and Theorem 2.3. The proof is based on some pointwise estimates on the derivative, which are the content of next two results. 
Proposition 3.5. Let $k$ be a positive integer, let $\alpha \in(0,1]$, let $(a, b) \subseteq \mathbb{R}$ be an interval, let $f:(a, b) \rightarrow \mathbb{R}$ be a continuous function, and let $g \in C^{k, \alpha}((a, b))$.

Let us assume that $f$ and $g$ satisfy (2.2), and that $g(x) \cdot g^{\prime}(x) \neq 0$ for every $x \in(a, b)$. Let us set for simplicity

$$
H:=\operatorname{Höld}_{\alpha}\left(g^{(k)},(a, b)\right), \quad \quad\left\|g^{\prime}\right\|_{\infty}:=\left\|g^{\prime}\right\|_{L^{\infty}((a, b))} .
$$

Then we have the following conclusions.

(1) There exists a constant $C_{1}(k)$, depending only upon $k$, such that for every $x \in$ $(a, b)$ we have that

$$
\left|f^{\prime}(x)\right|^{k+\alpha} \leq C_{1}(k) \max \left\{H,\left\|g^{\prime}\right\|_{\infty} \cdot\left[\frac{b-a}{(x-a)(b-x)}\right]^{k+\alpha-1}\right\} .
$$

(2) Let $p$ be defined by (2.5). Then there exists a constant $C_{2}(k)$, depending only upon $k$, such that

$$
\left\|f^{\prime}\right\|_{p, w,(a, b)} \leq C_{2}(k) \max \left\{H^{1 /(k+\alpha)} \cdot(b-a)^{1 / p},\left\|g^{\prime}\right\|_{\infty}^{1 /(k+\alpha)}\right\} .
$$

Proof. Let us fix any point $x_{0} \in(a, b)$, and let $d:=\min \left\{x_{0}-a, b-x_{0}\right\}$. We can apply Proposition 3.4 to the function $v(x):=g(x)$ in the interval $\left(x_{0}-d, x_{0}+d\right)$. We obtain that

$$
\left|f^{\prime}\left(x_{0}\right)\right|^{k+\alpha} \leq \frac{\left|g^{\prime}\left(x_{0}\right)\right|^{k+\alpha}}{\left|g\left(x_{0}\right)\right|^{k+\alpha-1}} \leq C(k) \max \left\{H, \frac{\left|g^{\prime}\left(x_{0}\right)\right|}{d^{k+\alpha-1}}\right\} .
$$

Since

$$
\frac{1}{d^{k+\alpha-1}} \leq\left[\frac{b-a}{\left(x_{0}-a\right)\left(b-x_{0}\right)}\right]^{k+\alpha-1},
$$

conclusion (3.8) easily follows.

Let us consider now statement (2). From (3.8) and definition (2.5) of $p$, we have that $\left|f^{\prime}(x)\right| \leq\left[C_{1}(k)\right]^{1 /(k+\alpha)} \max \left\{\psi_{1}(x), \psi_{2}(x)\right\}$, with

$$
\psi_{1}(x):=H^{1 /(k+\alpha)}, \quad \quad \psi_{2}(x):=\left\|g^{\prime}\right\|_{\infty}^{1 /(k+\alpha)} \cdot \frac{(b-a)^{1 / p}}{[(x-a)(b-x)]^{1 / p}} .
$$

From statements (2) through (4) of Lemma 3.1 we easily obtain (3.9).

Proposition 3.6. Let $k$ be a positive integer, let $\alpha \in(0,1]$, let $(a, b) \subseteq[c, d] \subseteq \mathbb{R}$ be an open and a closed interval, let $f:(a, b) \rightarrow \mathbb{R}$ be a continuous function, and let $g \in C^{k}([c, d])$. Let us assume that

(i) $f$ and $g$ satisfy (2.2),

(ii) $g(x) \cdot g^{\prime}(x) \neq 0$ for every $x \in(a, b)$, 
(iii) the $k$-th derivative $g^{(k)}(x)$ is $\alpha$-Hölder continuous in $(c, d)$ (hence also in $[c, d])$,

(iv) for every $h=1, \ldots, k$ there exists $x_{h} \in[c, d]$ such that $g^{(h)}\left(x_{h}\right)=0$.

Let $p$ be defined by (2.5), and let $C_{2}(k)$ the the constant in (3.9). Then we have that

$$
\left\|f^{\prime}\right\|_{p, w,(a, b)} \leq C_{2}(k)\left[\operatorname{Höld}_{\alpha}\left(g^{(k)},(c, d)\right)\right]^{1 /(k+\alpha)} \cdot(d-c)^{1 / p} .
$$

Proof. Let us set for simplicity $H:=\operatorname{Höld}_{\alpha}\left(g^{(k)},(c, d)\right)$. Due to assumptions (i), (ii), and (iii), we can apply Proposition 3.5 and obtain estimate (3.9). Due to assumptions (iii) and (iv), we can estimate in $[c, d]$ all derivatives of $g$ up to order $k$. For every $h=1, \ldots, k$ we obtain that

$$
\left|g^{(h)}(x)\right| \leq H \cdot(d-c)^{k+\alpha-h} \quad \forall x \in[c, d]
$$

(formally one should argue by induction on $k-h$ ), and in particular

$$
\left\|g^{\prime}\right\|_{L^{\infty}((a, b))} \leq\left\|g^{\prime}\right\|_{L^{\infty}((c, d))} \leq H \cdot(d-c)^{k+\alpha-1} .
$$

Plugging this inequality into (3.9), and estimating $(b-a)$ with $(d-c)$, we easily obtain (3.10).

Proof of Theorem 2.2. Let us consider the open set

$$
\Omega_{0}:=\{x \in(a, b): f(x) \neq 0\}=\{x \in(a, b): g(x) \neq 0\} .
$$

From (2.2) it is easy to see that $f \in C^{1}\left(\Omega_{0}\right)$ (or better $f \in C^{k}\left(\Omega_{0}\right)$ ), and

$$
\Omega_{1}:=\left\{x \in \Omega_{0}: f^{\prime}(x) \neq 0\right\}=\left\{x \in \Omega_{0}: g^{\prime}(x) \neq 0\right\} .
$$

Let $\mathcal{C}$ denote the (finite or countable) set of connected components of $\Omega_{1}$. Each $J \in \mathcal{C}$ is an open interval of the form $\left(a_{J}, b_{J}\right)$, so that

$$
\Omega_{1}=\bigcup_{J \in \mathcal{C}} J=\bigcup_{J \in \mathcal{C}}\left(a_{J}, b_{J}\right) .
$$

Thanks to Lemma 3.2 and statement (5) of Lemma 3.1 we have that

$$
\left\|f^{\prime}\right\|_{p, w,(a, b)}^{p}=\left\|f^{\prime}\right\|_{p, w, \Omega_{0}}^{p}=\left\|f^{\prime}\right\|_{p, w, \Omega_{1}}^{p} \leq \sum_{J \in \mathcal{C}}\left\|f^{\prime}\right\|_{p, w, J}^{p} .
$$

It is therefore enough to show that the right-hand side of (3.12) is estimated by the right-hand side of (2.6), raised to the power of $p$, for a suitable constant $C(k)$ depending only upon $k$. To this end, we divide the connected components of $\Omega_{1}$ into two disjoint classes $\mathcal{C}_{0}$ and $\mathcal{C}_{1}$. 
Partitioning connected components. Let $\mathcal{C}_{0} \subseteq \mathcal{C}$ be the set of connected components $J \in \mathcal{C}$ for which $\left(a_{J}, b_{J}\right)$ is such that either $a_{J}=a$ or

$$
\left|\left[b_{J}, b\right) \backslash \Omega_{1}\right| \leq 2 k,
$$

where vertical bars denote the number of elements of a set, and let $\mathcal{C}_{1}:=\mathcal{C} \backslash \mathcal{C}_{0}$. If $(a, b) \backslash \Omega_{1}$ is a finite set, then $\mathcal{C}$ is finite and $\mathcal{C}_{0}$ contains the leftmost and the $(2 k+1)$ rightmost connected components of $\Omega_{1}$, or $\mathcal{C}_{0}=\mathcal{C}$ if $|\mathcal{C}| \leq 2 k+1$.

If $(a, b) \backslash \Omega_{1}$ is not finite, then $\mathcal{C}_{0}$ contains the connected component of $\Omega_{1}$ with $a_{J}=a$ (if it exists), and the rightmost $h$ connected components of $\Omega_{1}$, where $h$ is the largest integer less than or equal to $(2 k+1)$ such that the rightmost $h$ connected components of $\Omega_{1}$ exist and their right-hand endpoints are not accumulation points of $(a, b) \backslash \Omega_{1}$.

Note that it may happen that $a$ and $b$ themselves are accumulation points of $(a, b) \backslash \Omega_{1}$, and in this case $\mathcal{C}_{0}$ is empty. It is also possible that $|\mathcal{C}|=2 k+2$ but $\left|\mathcal{C}_{0}\right|<2 k+2$, because it could happen that $(a, b) \backslash \Omega_{1}$ contains an interval.

What is important is that in any case we have that $\left|\mathcal{C}_{0}\right| \leq 2 k+2$.

Absolute continuity in the union of $\mathcal{C}_{0}$-components. Let us apply Proposition 3.5 in a connected component $J \in \mathcal{C}_{0}$. We obtain that

$$
\begin{aligned}
\left\|f^{\prime}\right\|_{p, w, J}^{p} & \left.\leq\left[C_{2}(k)\right]^{p} \max \left\{\left[\operatorname{Höld}_{\alpha}\left(g^{(k)}, J\right)\right)\right]^{p /(k+\alpha)} \cdot\left(b_{J}-a_{J}\right),\left\|g^{\prime}\right\|_{L^{\infty}(J)}^{p /(k+\alpha)}\right\} \\
& \leq\left[C_{2}(k)\right]^{p} \max \left\{\left[\operatorname{Höld}_{\alpha}\left(g^{(k)},(a, b)\right)\right]^{p /(k+\alpha)} \cdot(b-a),\left\|g^{\prime}\right\|_{L^{\infty}((a, b))}^{p /(k+\alpha)}\right\} .
\end{aligned}
$$

Summing over all $J \in \mathcal{C}_{0}$, and recalling that $\left|\mathcal{C}_{0}\right| \leq 2 k+2$, we deduce that

$$
\begin{aligned}
\sum_{J \in \mathcal{C}_{0}}\left\|f^{\prime}\right\|_{p, w, J}^{p} \leq & (2 k+2) \cdot\left[C_{2}(k)\right]^{p .} \\
& \cdot \max \left\{\left[\operatorname{Höld}_{\alpha}\left(g^{(k)},(a, b)\right)\right]^{p /(k+\alpha)} \cdot(b-a),\left\|g^{\prime}\right\|_{L^{\infty}((a, b))}^{p /(k+\alpha)}\right\} .
\end{aligned}
$$

Expanding $\mathcal{C}_{1}$-components. We prove that to every $J \in \mathcal{C}_{1}$ we can associate a closed interval $\left[c_{J}, d_{J}\right]$ in such a way that the following three conditions are satisfied.

(P1) We have that $\left(a_{J}, b_{J}\right) \subseteq\left[c_{J}, d_{J}\right] \subseteq(a, b)$ for every $J \in \mathcal{C}_{1}$.

(P2) Every $x \in(a, b)$ lies in the interior of at most $(2 k+1)$ such intervals, namely

$$
\left|\left\{J \in \mathcal{C}_{1}: x \in\left(c_{J}, d_{J}\right)\right\}\right| \leq 2 k+1 .
$$

(P3) For every $J \in \mathcal{C}_{1}$, and every $h=1, \ldots, k$, there exists $x_{J, h} \in\left[c_{J}, d_{J}\right]$ such that $g^{(h)}\left(x_{J, h}\right)=0$ (note that $g$ is always defined in the closed interval $\left.\left[c_{J}, d_{J}\right]\right)$. 
To this end, for every $J \in \mathcal{C}_{1}$ we set

$$
D_{J}:=\left\{x \in\left[b_{J}, b\right):\left|\left[b_{J}, x\right] \backslash \Omega_{1}\right| \geq 2 k+1\right\} .
$$

Since $J \notin \mathcal{C}_{0}$, we have that (3.13) is false. It follows that $D_{J} \neq \emptyset$, so that we can define $c_{J}:=a_{J}$ and $d_{J}:=\inf D_{J}<b$.

Roughly speaking, if $(a, b) \backslash \Omega_{1}$ is a finite set, then $\left[c_{J}, d_{J}\right]$ is the closure of the union of $\left(a_{J}, b_{J}\right)$ and of the first $2 k$ connected components of $\Omega_{1}$ on its right. If $(a, b) \backslash \Omega_{1}$ is not finite, then we stop the union as soon as we find an accumulation point of $(a, b) \backslash \Omega_{1}$. For example, $d_{J}=b_{J}$ when $b_{J}$ is an accumulation point of $(a, b) \backslash \Omega_{1}$.

We have to show that properties (P1) through (P3) are satisfied. This is trivial for (P1) because $c_{J}=a_{J}>a$ (we recall that the connected component of $\Omega_{1}$ with $a_{J}=a$, if it exists, does not belong to $\mathcal{C}_{1}$ ), and $b_{J} \leq d_{J}<b$.

In order to prove (P2), let us assume by contradiction that (3.15) is false for some $x_{0} \in(a, b)$. Then $x_{0} \in\left(c_{J}, d_{J}\right)$ for at least $(2 k+2)$ connected components $J_{0}, J_{1}, \ldots, J_{2 k+1}$ in $\mathcal{C}_{1}$. Let us assume, without loss of generality, that components are named in such a way that $b_{J_{0}}<b_{J_{1}}<\ldots<b_{J_{2 k+1}}$.

On the one hand we have that $\left[b_{J_{0}}, b_{J_{2 k}}\right] \backslash \Omega_{1} \supseteq\left\{b_{J_{0}}, b_{J_{1}}, \ldots, b_{J_{2 k}}\right\}$, hence this set has at least $(2 k+1)$ elements. This means that $b_{J_{2 k}} \in D_{J_{0}}$, hence $b_{J_{2 k}} \geq$ $d_{J_{0}}>x_{0}$. On the other hand we have also that $x_{0}>c_{J_{2 k+1}}=a_{J_{2 k+1}} \geq b_{J_{2 k}}$, which gives a contradiction.

It remains to prove (P3). Let us take any $x \in D_{J}$. Then $\left[b_{J}, x\right]$ contains at least $(2 k+1)$ points which are not in $\Omega_{1}$, hence at least $(2 k+1)$ points where either $g$ vanishes or $g^{\prime}$ vanishes. As a consequence, $\left[b_{J}, x\right]$ contains either at least $(k+1)$ points where $g$ vanishes, or at least $k$ points where $g^{\prime}$ vanishes. In the first case Rolle's Theorem implies the existence of at least $k$ points where $g^{\prime}$ vanishes, so actually we are always in the second case.

Applying Rolle's Theorem again, we obtain that $\left[b_{J}, x\right]$ contains at least $(k-1)$ points where $g^{\prime \prime}$ vanishes, at least $(k-2)$ points where $g^{\prime \prime \prime}$ vanishes, and so on, up to find at least one point where $g^{(k)}$ vanishes. We have thus proved that all derivatives of $g$ up to order $k$ vanish at least once in $\left[b_{J}, x\right]$. Since they are continuous functions, the same is true in $\left[b_{J}, d_{J}\right]$, hence also in $\left[c_{J}, d_{J}\right]$. This completes the proof of (P3).

Absolute continuity in the union of $\mathcal{C}_{1}$-components. Let $J \in \mathcal{C}_{1}$, and let $\left[c_{J}, d_{J}\right]$ be the interval defined in the previous paragraph. Due to (P1) and (P3) we can apply Proposition 3.6 in the intervals $\left(a_{J}, b_{J}\right) \subseteq\left[c_{J}, d_{J}\right]$. We obtain that

$$
\left\|f^{\prime}\right\|_{p, w, J}^{p} \leq\left[C_{2}(k)\right]^{p}\left[\operatorname{Höld}_{\alpha}\left(g^{(k)},\left(c_{J}, d_{J}\right)\right)\right]^{p /(k+\alpha)} \cdot\left(d_{J}-c_{J}\right) .
$$

Estimating the Hölder constant in $\left(c_{J}, d_{J}\right)$ with the Hölder constant in $(a, b)$, and summing over all $J \in \mathcal{C}_{1}$, we deduce that

$$
\sum_{J \in \mathcal{C}_{1}}\left\|f^{\prime}\right\|_{p, w, J}^{p} \leq\left[C_{2}(k)\right]^{p}\left[\operatorname{Höld}_{\alpha}\left(g^{(k)},(a, b)\right)\right]^{p /(k+\alpha)} \cdot \sum_{J \in \mathcal{C}_{1}}\left(d_{J}-c_{J}\right) .
$$


Now we estimate the series in the right-hand side through a "geometric double counting" argument. Let $\chi_{\left(c_{J}, d_{J}\right)}(x)$ be 1 if $x \in\left(c_{J}, d_{J}\right)$, and 0 otherwise. Due to (3.15) we have that

$$
\begin{aligned}
\sum_{J \in \mathcal{C}_{1}}\left(d_{J}-c_{J}\right) & =\sum_{J \in \mathcal{C}_{1}} \int_{a}^{b} \chi_{\left(c_{J}, d_{J}\right)}(x) d x \\
& =\int_{a}^{b}\left(\sum_{J \in \mathcal{C}_{1}} \chi_{\left(c_{J}, d_{J}\right)}(x)\right) d x \leq(2 k+1)(b-a),
\end{aligned}
$$

hence

$$
\sum_{J \in \mathcal{C}_{1}}\left\|f^{\prime}\right\|_{p, w, J}^{p} \leq(2 k+1)\left[C_{2}(k)\right]^{p}\left[\operatorname{Höld}_{\alpha}\left(g^{(k)},(a, b)\right)\right]^{p /(k+\alpha)} \cdot(b-a) .
$$

From (3.14) and (3.16) we easily obtain (2.6).

Proof of Theorem 2.3. Let us assume, without loss of generality, that $\Omega^{\prime}:=\left(a_{1}, b_{1}\right) \times$ $\ldots \times\left(a_{n}, b_{n}\right)$ is a rectangle. From statement (1) of Lemma 3.1 we have that

$$
\|\nabla f\|_{p, w, \Omega^{\prime}} \leq\left\|\sum_{m=1}^{n}\left|f_{x_{m}}\right|\right\|_{p, w, \Omega^{\prime}} \leq n \sum_{m=1}^{n}\left\|f_{x_{m}}\right\|_{p, w, \Omega^{\prime}}
$$

Thus we can limit ourselves to showing that all partial derivatives of $f$ lie in $L_{w}^{p}\left(\Omega^{\prime}\right)$. Without loss of generality, we prove this fact in the case $m=1$. Let $y:=\left(x_{2}, \ldots, x_{n}\right)$ denote the vector of the remaining variables, and let $\Omega^{\prime \prime}:=$ $\left(a_{2}, b_{2}\right) \times \ldots \times\left(a_{n}, b_{n}\right)$. Then for every $M \geq 0$ we have that

$$
\operatorname{meas}\left\{x \in \Omega^{\prime}:\left|f_{x_{1}}(x)\right|>M\right\}=\int_{\Omega^{\prime \prime}} \operatorname{meas}\left\{x_{1} \in\left(a_{1}, b_{1}\right):\left|f_{x_{1}}\left(x_{1}, y\right)\right|>M\right\} d y \text {. }
$$

For every fixed $y \in \Omega^{\prime \prime}$, the measure in the integral involves only the function $x \rightarrow f(x, y)$, which is a function of one real variable. Therefore the measure can be estimated with the aid of Theorem 2.2 in terms of the function of one real variable $x \rightarrow g(x, y)$, which for simplicity we denote by $g(\cdot, y)$. We obtain that

$$
\begin{aligned}
& \text { meas }\left\{x_{1} \in\left(a_{1}, b_{1}\right):\left|f_{x_{1}}\left(x_{1}, y\right)\right|>M\right\} \leq \frac{1}{M^{p}}\left\|f_{x_{1}}(\cdot, y)\right\|_{p, w,\left(a_{1}, b_{1}\right)}^{p} \\
& \leq \frac{[C(k)]^{p}}{M^{p}} \max \left\{\left[\operatorname{Höld}_{\alpha}\left(D_{x_{1}}^{k} g(\cdot, y),\left(a_{1}, b_{1}\right)\right)\right]^{p /(k+\alpha)}\left(b_{1}-a_{1}\right),\left\|g_{x_{1}}(\cdot, y)\right\|_{L^{\infty}\left(\left(a_{1}, b_{1}\right)\right)}^{p /(k+\alpha)}\right\} \\
& \leq \frac{[C(k)]^{p}}{M^{p}} \max \left\{\left[\operatorname{Höld}_{\alpha}\left(g^{(k)}, \Omega\right)\right]^{p /(k+\alpha)} \cdot\left(b_{1}-a_{1}\right),\|\nabla g\|_{L^{\infty}(\Omega)}^{p /(k+\alpha)}\right\} .
\end{aligned}
$$


Therefore we can estimate the left-hand side of (3.17) with the right-hand side of (3.18) multiplied by the $(n-1)$-dimensional measure of $\Omega^{\prime \prime}$.

Thus we obtain that

$$
\begin{aligned}
\left\|f_{x_{1}}\right\|_{p, w, \Omega^{\prime}} & =\sup _{M \geq 0} M \cdot\left[\operatorname{meas}\left\{x \in \Omega^{\prime}:\left|f_{x_{1}}(x)\right|>M\right\}\right]^{1 / p} \\
& \leq C(k) \max \left\{\left[\operatorname{Höld}_{\alpha}\left(g^{(k)}, \Omega\right)\right]^{1 /(k+\alpha)} \cdot\left|\Omega^{\prime}\right|^{1 / p},\|\nabla g\|_{L^{\infty}(\Omega)}^{p /(k+\alpha)} \cdot\left|\Omega^{\prime \prime}\right|^{1 / p}\right\},
\end{aligned}
$$

where $\left|\Omega^{\prime}\right|$ and $\left|\Omega^{\prime \prime}\right|$ are the $n$-dimensional measure of $\Omega^{\prime}$, and the $(n-1)$-dimensional measure of $\Omega^{\prime \prime}$, respectively.

This is enough to complete the proof.

\section{Examples and open problem}

Example 4.1. Let us consider the function $v(x):=\sin ^{2} x+e^{-x^{2}}$. Then $v \in$ $C^{\infty}(\mathbb{R})$, and all its derivatives are bounded in $\mathbb{R}$, which means that $v \in C^{k, \alpha}(\mathbb{R})$ for every admissible value of $k$ and $\alpha$. Moreover $v(x)>0$ for every $x \in \mathbb{R}$. Let us consider the sequence $x_{n}:=2 \pi n+n^{-1}$. It is not difficult to see that

$$
v\left(x_{n}\right) \sim \frac{1}{n^{2}}, \quad v^{\prime}(x) \sim \frac{2}{n} \quad \text { as } n \rightarrow+\infty,
$$

hence the ratio (2.4) is not bounded whenever $k \geq 2$ and $\alpha>0$.

This example shows that the conclusion of Theorem 2.1 is false when $v^{\prime}(x)$ is a sign changing function (as in this example).

Example 4.2. Let $\varphi \in C^{\infty}(\mathbb{R})$ be a function such that $\varphi(x)=1$ for every $x \leq 0$, $\varphi(x)=0$ for every $x \geq 1$, and $\varphi^{\prime}(x)<0$ for every $x \in(0,1)$. We note that these conditions imply that all derivatives of $\varphi$ vanish in $x=0$ and $x=1$. Let us consider the following sequences

$$
\gamma_{n}:=e^{-(n+1)^{2}}, \quad a_{n}:=\gamma_{n}^{k+\alpha} \cdot\left|\log \gamma_{n}\right|, \quad \alpha_{n}:=\sum_{i=n+1}^{\infty} a_{i} .
$$

Some simple calculus shows that $\alpha_{n}$ is well defined (namely the series converges), and

$$
\lim _{n \rightarrow+\infty} \frac{\alpha_{n}}{a_{n}}=0
$$

Let $w: \mathbb{R} \rightarrow \mathbb{R}$ be defined by

$$
w(x):= \begin{cases}\alpha_{0}+a_{0} & \text { if } x \leq 0 \\ \alpha_{n}+a_{n} \varphi\left(\gamma_{n}^{-1}(x-n)\right) & \text { if } x \in\left[n, n+\gamma_{n}\right] \\ \alpha_{n} & \text { if } x \in\left[n+\gamma_{n}, n+1\right] .\end{cases}
$$


It is easy to see that $w \in C^{\infty}(\mathbb{R})$, and for every $x \in \mathbb{R}$ we have that $w(x)>0$ and $w^{\prime}(x) \leq 0$. Moreover for every $\beta \in(0,1]$ we have that

$$
w \in C^{k, \beta}(\mathbb{R}) \Longleftrightarrow \sup _{n \in \mathbb{N}} \frac{a_{n}}{\gamma_{n}^{k+\beta}}<+\infty
$$

hence $w \in C^{k, \beta}(\mathbb{R})$ for every $\beta<\alpha$, but not for $\beta=\alpha$.

Let us consider now the function $\psi(x):=\arctan e^{-\left(x-\gamma_{0} / 2\right)^{3}}$, and let us set $v(x):=w(x)+\psi(x)$. It is not difficult to see that $\psi \in C^{\infty}(\mathbb{R})$, its derivatives of any order are bounded in $\mathbb{R}$, and $\psi(x)>0$, hence also $v(x)>0$, for every $x \in \mathbb{R}$. Moreover we have that $\psi^{\prime}(x) \leq 0$ for every $x \in \mathbb{R}$, with equality if and only if $x=\gamma_{0} / 2$ (where $w^{\prime}(x)$ does not vanish), so that $v^{\prime}(x)<0$ for every $x \in \mathbb{R}$. Finally we have that $v \in C^{k, \beta}(\mathbb{R})$ for every $\beta<\alpha$, but not for $\beta=\alpha$. that

Let us consider now the sequence $z_{n}:=n+\gamma_{n} / 2$. It is not difficult to check

$$
\lim _{n \rightarrow+\infty} \frac{\psi\left(z_{n}\right)}{a_{n}}=0, \quad \lim _{n \rightarrow+\infty} \frac{\psi^{\prime}\left(z_{n}\right) \cdot \gamma_{n}}{a_{n}}=0 .
$$

Exploiting also (4.1), it follows that (up to numeric constants) we have that

$$
\begin{aligned}
& v\left(z_{n}\right)=a_{n} \varphi(1 / 2)+\alpha_{n}+\psi\left(z_{n}\right) \sim a_{n} \quad \text { as } n \rightarrow+\infty, \\
& v^{\prime}\left(z_{n}\right)=\frac{a_{n}}{\gamma_{n}} \varphi^{\prime}(1 / 2)+\psi^{\prime}\left(z_{n}\right) \sim \frac{a_{n}}{\gamma_{n}} \quad \text { as } n \rightarrow+\infty \text {. }
\end{aligned}
$$

This implies that the sequence

$$
\frac{\left|v^{\prime}\left(z_{n}\right)\right|^{k+\alpha}}{\left[v\left(z_{n}\right)\right]^{k+\alpha-1}} \sim \frac{a_{n}^{k+\alpha}}{\gamma_{n}^{k+\alpha}} \cdot \frac{1}{a_{n}^{k+\alpha-1}}=\frac{a_{n}}{\gamma_{n}^{k+\alpha}}=\left|\log \gamma_{n}\right|
$$

is unbounded, hence the ratio (2.4) is unbounded.

Example 4.3. Let $f:(-1,1) \rightarrow \mathbb{R}$ and $g:(-1,1) \rightarrow \mathbb{R}$ be defined by

$$
f(x):=|x|^{1 /(k+\alpha)}, \quad g(x):=x .
$$

All assumptions of Theorem 2.2 are satisfied (and $g$ is actually of class $C^{\infty}$ ), and $f^{\prime} \notin L^{p}((-1,1))$ when $p$ is defined by $(2.5)$. This shows that the summability of $f^{\prime}$ given by Theorem 2.2 is optimal.

Example 4.4. Let $\varphi \in C^{\infty}(\mathbb{R})$ be a function such that $\varphi(x)>0$ for every $x \in$ $(0,1)$, and $\varphi(x)=0$ elsewhere, so that all its derivatives vanish in $x=0$ and $x=1$. Let us assume also that $\varphi^{\prime}(x)>0$ for every $x \in(0,1 / 2)$, and $\varphi^{\prime}(x)<0$ for every $x \in(1 / 2,1)$, so that it is quite easy to estimate the total variation of $\varphi$ and all its roots. Let us consider the following sequences

$$
\gamma_{n}:=\frac{1}{(n+3) \log (n+3) \log ^{2}(\log (n+3))}, \quad \lambda_{n}:=\sum_{i=n}^{\infty} \gamma_{i}, \quad a_{n}:=\gamma_{n}^{k+\alpha} \cdot\left|\log \gamma_{n}\right| .
$$


Some simple calculus shows that $\lambda_{n}$ is well defined (namely the series converges). Let $g:\left(0, \lambda_{0}\right) \rightarrow \mathbb{R}$ be defined by

$$
g(x):=a_{n} \varphi\left(\frac{x-\lambda_{n+1}}{\lambda_{n}-\lambda_{n+1}}\right) \quad \forall x \in\left[\lambda_{n+1}, \lambda_{n}\right),
$$

and let $f(x):=[g(x)]^{1 /(k+\alpha)}$ be its $(k+\alpha)$-th root. that

It is easy to see that $g \in C^{\infty}\left(\left(0, \lambda_{0}\right)\right)$. Moreover for every $\beta \in(0,1]$ we have

$$
g \in C^{k, \beta}\left(\left(0, \lambda_{0}\right)\right) \Longleftrightarrow \sup _{n \in \mathbb{N}} \frac{a_{n}}{\gamma_{n}^{k+\beta}}<+\infty,
$$

hence $g \in C^{k, \beta}\left(\left(0, \lambda_{0}\right)\right)$ for every $\beta<\alpha$, but not for $\beta=\alpha$. Moreover the total variation of $f$ in $\left(0, \lambda_{0}\right)$ is given by

$$
T V\left(f,\left(0, \lambda_{0}\right)\right)=2 \sum_{n=0}^{\infty} a_{n}^{1 /(k+\alpha)} \cdot[\varphi(1 / 2)]^{1 /(k+\alpha)},
$$

hence

$$
f^{\prime} \in L^{1}\left(\left(0, \lambda_{0}\right)\right) \Longrightarrow f \in B V\left(\left(0, \lambda_{0}\right)\right) \Longrightarrow \sum_{n=0}^{\infty} a_{n}^{1 /(k+\alpha)}<+\infty
$$

(actually also the reverse implications hold true, but we don't need them). Now some simple calculus shows that

$$
\sum_{n=0}^{\infty} a_{n}^{1 /(k+\alpha)}=\sum_{n=0}^{\infty} \gamma_{n}\left|\log \gamma_{n}\right|^{1 /(k+\alpha)}=+\infty
$$

This means that $f(x)$ is not a bounded variation function in $\left(0, \lambda_{0}\right)$, and hence $f(x)$ is not even absolutely continuous in $\left(0, \lambda_{0}\right)$.

We conclude by recalling the main open problem in this field, namely the absolute continuity of roots of polynomials instead of roots of functions. We point out that the problem is open even in the case of a real root of a non-hyperbolic polynomial with real coefficients of class $C^{\infty}$.

Open problem. Let $k$ be a positive integer, let $(a, b) \subseteq \mathbb{R}$ be an interval, and let

$$
P(x):=x^{k+1}+a_{k}(t) x^{k}+\ldots+a_{1}(t) x+a_{0}(t)
$$

be a monic polynomial with complex valued coefficients $a_{i} \in C^{k, 1}((a, b))$. Let $z:(a, b) \rightarrow \mathbb{C}$ be a continuous function such that $P(z(t))=0$ for every $t \in(a, b)$.

Then $z^{\prime} \in L_{w}^{p}((a, b))$ for $p=1+1 / k$. 


\section{References}

[1] D. Alekseevsky, A. Kriegl, M. Losik and P. W. Michor, Choosing roots of polynomials smoothly, Israel J. Math. 105 (1998), 203-233.

[2] J. M. BONY, F. COLOMBINI and L. PERnAZZA, On square roots of class $C^{m}$ of nonnegative functions of one variable, Ann. Sc. Norm. Super. Pisa Cl. Sci. (5) 9 (2010), 635-644.

[3] M. D. BRONŠTE YN, Smoothness of roots of polynomials depending on parameters, Sibirsk. Mat. Zh. 20 (1979), 493-501, 690 (English translation: Siberian Math. J. 20 (1979), $347-$ $352(1980))$.

[4] F. Colombini, E. Jannelli and S. Spagnolo, Well-posedness in the Gevrey classes of the Cauchy problem for a nonstrictly hyperbolic equation with coefficients depending on time, Ann. Scuola Norm. Sup. Pisa Cl. Sci. (4) 10 (1983), 291-312.

[5] F. COLOMBINI and N. LERNER, Une procédure de Calderón-Zygmund pour le problème de la racine k-ième, Ann. Mat. Pura Appl. (4) 182 (2003), 231-246.

[6] F. COLOMBInI, N. ORRÙ and L. PeRnAZZA, On the regularity of the roots of hyperbolic polynomials, Israel J. Math. 191 (2012), 923-944.

[7] G. GLAESER, Racine carrée d'une fonction différentiable, Ann. Inst. Fourier (Grenoble) $\mathbf{1 3}$ (1963), 203-210.

[8] T. Kato, "A Short Introduction to Perturbation Theory for Linear Operators", SpringerVerlag, New York-Berlin, 1982.

[9] A. KRIEGL, M. LOSIK and P. W. MICHOR, Choosing roots of polynomials smoothly. II, Israel J. Math. 139 (2004), 183-188.

[10] T. MANDAI, Smoothness of roots of hyperbolic polynomials with respect to one-dimensional parameter, Bull. Fac. Gen. Ed. Gifu Univ. 21 (1985), 115-118.

[11] A. RAINER, Perturbation of complex polynomials and normal operators, Math. Nachr. 282 (2009), 1623-1636.

[12] S. SPAGNOLO, On the absolute continuity of the roots of some algebraic equations, Ann. Univ. Ferrara Sez. VII (N.S.), 45 (1999) suppl., 327-337.

[13] S. TARAma, On the Lemma of Colombini, Jannelli and Spagnolo, Mem. of the Faculty of Engineering Osaka City Univ. 41 (2000), 111-115.

[14] S. TARAMA, Note on the Bronshtein theorem concerning hyperbolic polynomials, Sci. Math. Jap. 63 (2006), 247-285.

[15] S. WAKABAYASHI, Remarks on hyperbolic polynomials, Tsukuba J. Math. 10 (1986), 17 28.

Università degli Studi di Pisa

Dipartimento di Matematica

Largo Bruno Pontecorvo, 5

56127 Pisa, Italia

ghisi@dm.unipi.it

m.gobbino@dma.unipi.it 